\title{
Experimental and numerical investigation of mode II failure behavior evaluation using three point bend, end notched flexure test
}

\author{
R. Nikhil ${ }^{*}$, S. Shivakumar ${ }^{1}$, Kallol Anupama ${ }^{1}$ and Shettar Manjunath ${ }^{2}$ \\ ${ }^{1}$ Department of Mechanical Engineering, KLS Gogte Institute of Technology, Belagavi, India \\ ${ }^{2}$ Department of Mechanical Engineering, Manipal Institute of Technology, Manipal, India
}

\begin{abstract}
In the present paper the primary task is the study involving calculation of elastic properties of the composite from the individual properties of the E-glass fiber ( $650 \mathrm{GSM}$ ) and the properties of resin LY 556 with Hardener HY951. The properties of varying volumetric ratio of fiber are obtained from calculation of the properties by using rule of mixtures. Experimentally validating the theoretical and numerical approaches by comparing the load-displacement response and crack paths observed in large scale bridged crack propagation in laminated fiberreinforced composites specimens. An effort is being made to develop a numerical framework for cohesive crack propagation and demonstrating its effectiveness by simulating failure through crack propagation in materials with complex microstructure like fiber reinforced composites. Experimentally validating the theoretical and numerical approaches by comparing the load-displacement response and crack paths observed in large scale bridged crack propagation in laminated fiber-reinforced composites specimens.
\end{abstract}

\section{Introduction}

The introduction of fiber-reinforced composites has been a major step in the evolution of structural frames. Limited attention has been paid to modeling the fracture of crack-like flaws in pultruded composite systems, fracture mechanics has previously been advocated and applied to thin-section laminated composites. Sih et al.[1] used a complex variable approach to derive the general equations of crack-tip stress fields in anisotropic bodies. Finite element analysis using the virtual crack closure technique [2-3] has been used to determine the strain energy release rate and relate it to the SIF. In an attempt to use existing stress intensity factor (SIF) solutions derived for isotropic conditions, Mandell et al. [4] used hybrid FE analysis to show that the SIF can be dependent on the degree of material anisotropy for various specimen geometries. The SIF change between isotropic and anisotropic cases was relatively constant for varying crack lengths in a given geometry. The material constants were found to have an effect on the crack-tip stresses. Boa et al. [5-6]

${ }^{*}$ Corresponding author: nikhilr@git.edu 
used an orthotropy rescaling technique to fit formulae into numerical calibrations to quantify the effect of material orthotropy on notched bars, delaminated beams, and hybrid sandwiches.

Stress based failure criteria were proposed to determine the strength of notched composites. Some of these criteria were evaluated point-wise while others were determined using average variables or characteristic lengths. Whitney and Nussimer [7] proposed a failure criterion using the average stress distribution calculated from the edge of the hole to a given characteristic distance. The distance from the edge was considered as a material property since it did not depend on the size of the notch. A second related criterion was proposed where failure is assumed to occur when the stress, at a distance away from the notch, reaches the strength of the unnotched material. The proposed criteria were applied to graphite / epoxy and glass / epoxy laminated composites with straight cracks and were used to explain the reduction in strength of composites with larger hole sizes. Sih et al. [8] proposed a strain energy density failure criterion to predict fracture in unidirectional composites subject to off-axis loading. Despite limitations, such as the violation of selfsimilar crack growth in various laminates, Linear Fracture Mechanics (LFM) has been applied to study failure in different composite systems [9-11] used a stress intensity factor (SIF) calculated based on amodified crack size. The larger effective crack length was determined by equating the intense energy region ahead of the crack to a material dependent characteristic length.

The paper starts with general theory regarding the determining of crack behaviour due to propagation and lifetime estimation. A numerical solution is conducted in ANSYS, and a process of programming due to obtain generals numerical solutions for crack trajectories and lifetime estimations is accomplished. In order to verify the numerical solutions from ANSYS, experimental results are taken into account for benchmarking. The methodology intended to be followed includes:

Phase 1: Overview of fractography and stress concentration factor

Phase 2: Development of elasto-plastic FEA model

Phase 3: Experimental studies

Phase 4: Comparison between FE and Experimental studies.

\section{Experimental study}

The experimental study involves calculation of elastic properties of the composite from the individual properties of the E-glass fiber (650 GSM) and the properties of resin LY 556 with Hardener HY951. The properties of varying volumetric ratio of fiber are obtained from calculation of the properties by using rule of mixtures.

The three points bending flexural test gives values for the modulus of elasticity in bending Ef, flexural stress $\sigma_{\mathrm{f}}$, flexural strain $\varepsilon_{\mathrm{f}}$ and the flexural stress-strain response of the material. The main advantage of a three point flexural test is the ease of the specimen preparation and testing. However, this method has also some disadvantages: the results of the testing method are sensitive to specimen and loading geometry and strain rate.

Fig. 2.1 shows the experimental setup of the specimen in UTM. The testing procedure for conducting the test usually involves a specified test fixture that can be movable to adjust according to the required support span needed on a universal testing machine where loading is done uniformly and at constant rate with respect to time. Details of the specimen preparation, conditioning, and conduct affect the test results as it could lead to improper binding of laminates thus three specimens of same volumetric ratio are taken and their average peak load is taken. The sample is placed on two supporting pins a set distance apart and a third loading pin is lowered from above at a constant rate until sample failure. 

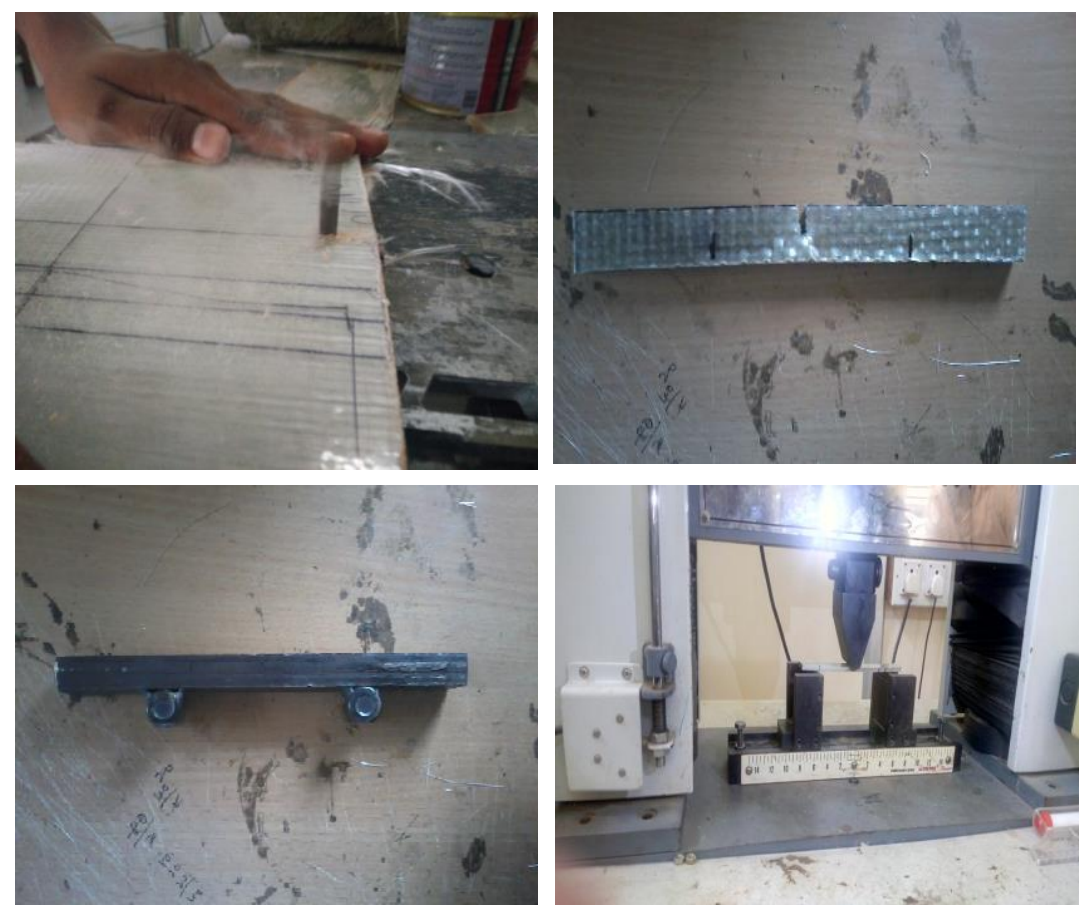

Fig. 2.1 Specimen preparation \& experimental setup for fabrication and testing on UTM

Flexural stress given in following equation and is calculated by

$$
\sigma_{\mathrm{f}}=\frac{3 \mathrm{FL}}{2 \mathrm{bd}^{2}}
$$

The experimental three point bending test by compliance calibration method is conducted on the ENF specimens to obtain peak critical load Pc at which the specimen fails and mode II crack propagates accompanied by sudden decrease in load in the load vs deflection graph. Then experiment is conducted for all specimens of varying volumetric ratio of fiber-resin and also on uncracked specimens.

For these values for $0.4,0.5,0.6$ fiber volume ratio the $\mathrm{G}_{\text {IIC }}$ is calculated

$$
G_{\text {IIC }}=\frac{9 \mathrm{P}^{2} \mathrm{Ca}^{2}}{2 \mathrm{w}\left(2 \mathrm{~L}^{3}+3 \mathrm{a}^{3}\right)}
$$

The compliance (C) can be found by Equation

$$
\mathrm{C}=\frac{2 \mathrm{~L}^{3}+3 \mathrm{a}^{3}}{8 \mathrm{Ewh}^{3}}
$$

\section{Cohesive zone modelling (CZM)}

The ENF specimen is analyzed by CZM method to obtain SERR By using the peak load from the experimental values. These numerical values are compared with experimental values. Fig. 3.1 shows that CZM analysis is done on ENF specimen and Von Mises stress is obtained for different volumetric ratio of fiber, with peak load from experimental values taken and input as point load at the centre of specimen in direction being perpendicular to fibers along the length of specimen. 

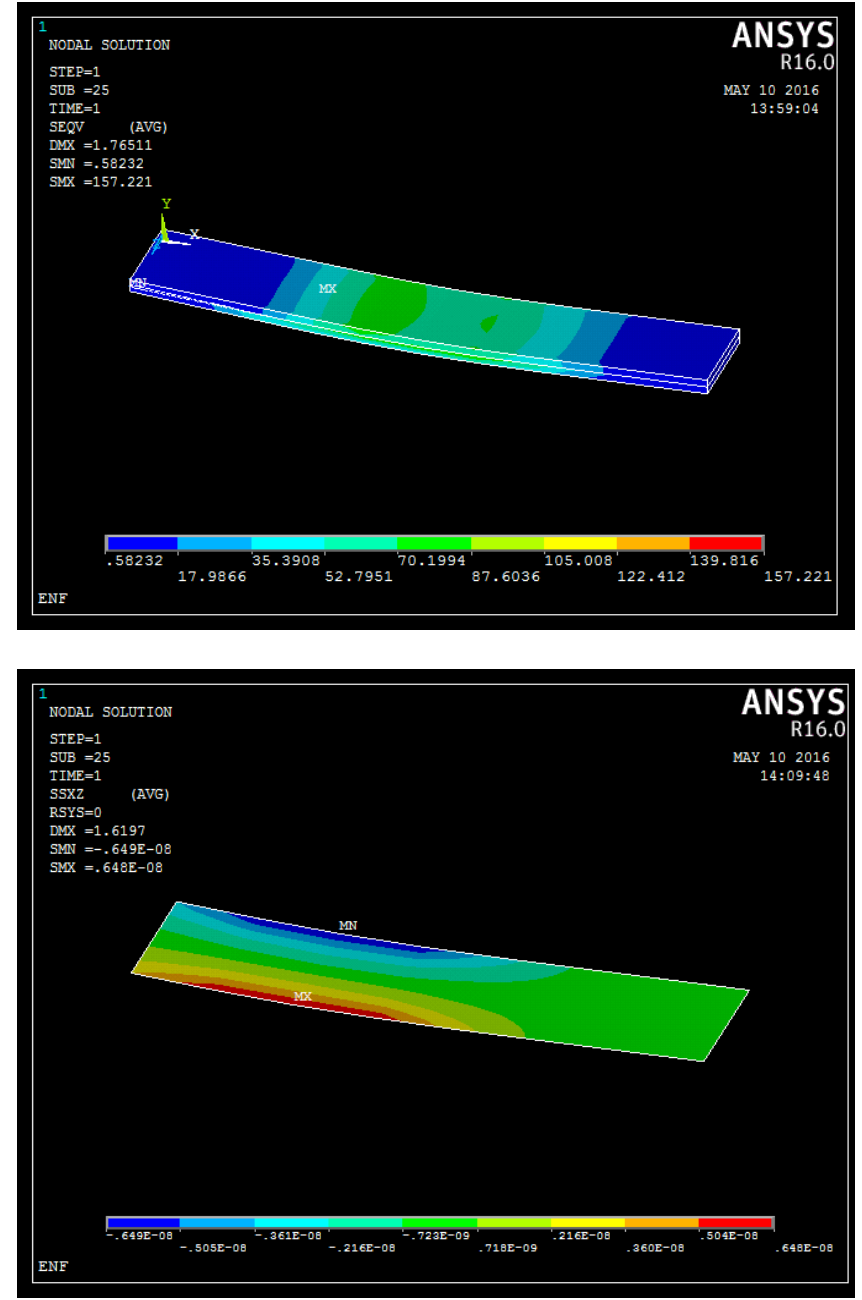

Fig. 3.1 Von- Misses Stress and displacement by CZM approach

Now, comparing the results from experimental and CZM approach shows that the values of SERR obtained from CZM has slightly higher values than experimental values of SERR (Fig. 3.2) and the difference of SERR for 0.5 fiber volume fraction is slightly less when compared to other values.

Table 3.1 Strain Energy Release Rate (SERR) of Experimental and CZM

\begin{tabular}{|c|c|c|c|c|}
\hline $\begin{array}{c}\text { Volume } \\
\text { fraction of } \\
\text { fiber }\end{array}$ & $\begin{array}{c}\text { Average peak } \\
\text { load with } \\
\text { crack } \\
(\mathrm{N})\end{array}$ & $\begin{array}{c}\text { Average peak } \\
\text { load without } \\
\text { crack } \\
(\mathrm{N})\end{array}$ & $\begin{array}{c}\text { Strain Energy } \\
\text { Release Rate - } \\
\text { Experimental } \\
\text { Method } \\
(\mathrm{J} / \mathrm{mm} 2)\end{array}$ & $\begin{array}{c}\text { Strain Energy } \\
\text { Release Rate - } \\
\text { CZM } \\
\text { Analysis } \\
(\mathrm{J} / \mathrm{mm} 2)\end{array}$ \\
\hline 0.4 & 2324.7 & 3379 & 0.74 & 1.15 \\
\hline 0.5 & 2617.3 & 3547 & 1.59 & 1.78 \\
\hline 0.6 & 3172.2 & 3726 & 2.10 & 2.37 \\
\hline
\end{tabular}




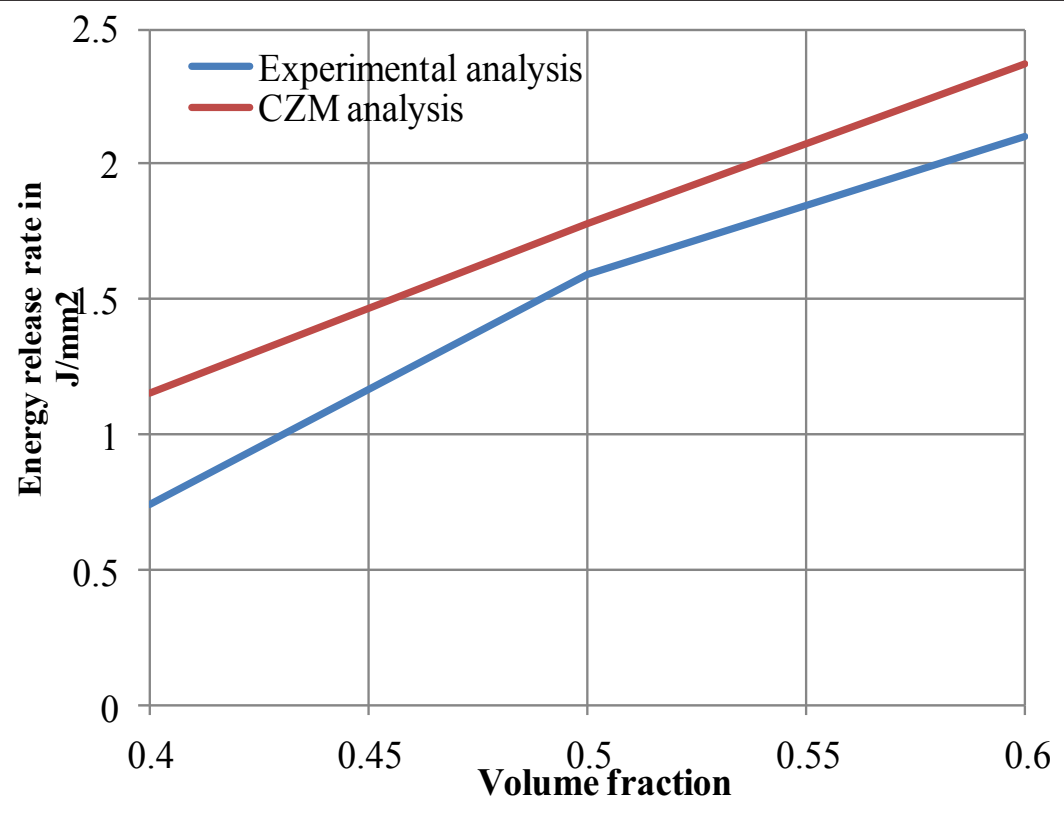

Fig. 3.2 Comparision of energy release rate of experimental and CZM approach

\subsection{Fracture surface}

Fig. 3.3 (a) and 3.3 (b-c) shows the fracture surfaces of un-notched epoxy/ glass composites and notched epoxy/glass composites respectively. Un-notched composites show lesser degree of fibre pullout (Fig. 3.3(a)), which is due to the delay in crack initiation and higher interfacial strength. The notched composites showed high fibre breakage due to initial damage and lesser interfacial strength. The resin-fibre bonds have broken and lumps of resin are observed in amorphous form, Fig. 3.3 (b-d)
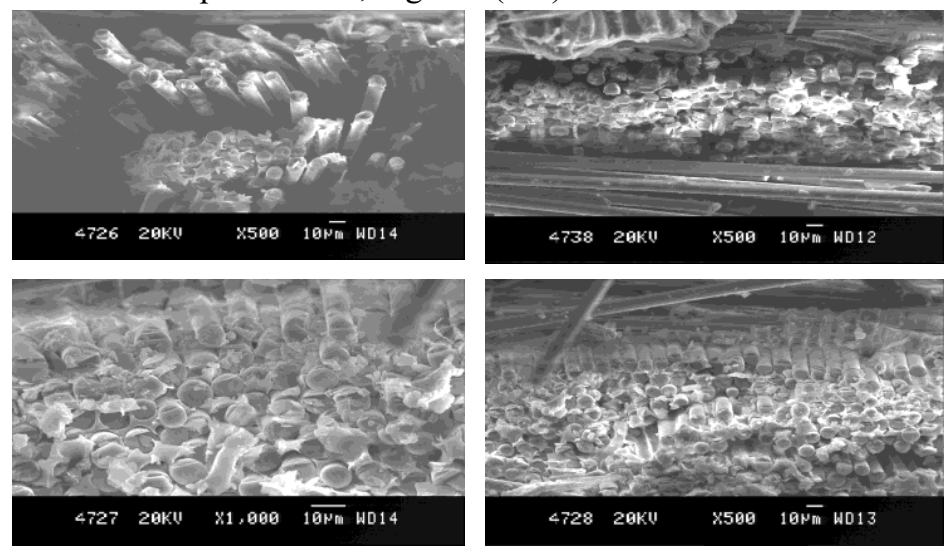

Fig. 3.3: Tensile fracture surfaces of a) unmodified glass laminates and b) modified glass laminates 


\section{Summary of results}

$>$ From the experimental analysis performed it shows that the delamination method of crack propagation is mainly dominated by inter-laminar delamination between the layers and the crack propagates exactly along the middle layer where the initial crack was initiated, intra-laminar delamination, buckling of fibers and fiber effects like hygrothermal, moisture absorption and temperature effects do not contribute significantly to contribute (i.e. its effects are neglected during testing).

$>$ Delaminations also occur due to improper binding, non uniform distribution of resin, presence of foreign particles ,absorption of moisture, non uniform compaction during pressing of laminate and curing of resin along the layers.

$>$ During experimentation there is variation and load fluctuation during peak loads due buckling of fibers and after the peak load accompanied by loud sound, the load carrying capacity decreases indicated by steep fall in curve (load vs displacement ).

$>$ For varying volumetric composition the increasing content of fiber in ENF specimens have more load carrying capacity thereby having greater SERR and thus offers more resistance to crack propagation as compared to lower percentage of fibers. But too high fiber percentage $(>75 \%)$ means more brittle nature and less bonding due low resin content thereby crack propagates easily.

$>$ Comparison of numerical results with available experimental ones showed that the CZM method, in spite of simplicity, cannot predict the propagation behaviour in unidirectional ENF specimen with fiber bridging very well. Hence a method such as Virtual Crack Closure Technique (VCCT) can also be used to study mode II failure behavior evaluation using three point bend, end notched flexure test

\section{References}

1. Sih. G. C., Chen E. P., Huang S. L., and Mc.Quillen E. J., J. Comp. Mate., vol. 9, pp. 167- (1975).

2. Raju I. S., Engg. Frac. Mech., vol. 28, pp. 251-274, (1987).

3. Krueger R., NASA/CR-2002-211628, ICASE Report no. 2002-10, 2002.

4. Mandell J. F., McGarry F. J., Wang S. S., and Im J., J of Comp. Mate., vol. 8, pp. 106116, (1974).

5. Boa G., Ho S., Suo Z., and Fan B., Int. J. of Solids \& Struc., vol. 29, no. 9, pp. $1105-$ 1116, (1992).

6. Boa G., Ho S., Suo Z., and Fan B., Corrigenda, Int. J. Solids \& Struc., vol. 29, no. 16, pp. 2115, (1992).

7. Whitney J. M., Pagano, NJ editors. Interlaminar response of composite materials. Amsterdam, Elsevier, pp. 161-250, (1989).

8. Sih G. C., Paris, P. C., Irwin, G. R., Int. J. of Frac. Mech., vol. 1, no. 3, pp. 189-203, (1965).

9. Konish Jr., H. J., Swedlow, J. L. Cruse, T. A., AIAA Journal, vol. 11, no. 3, pp.40-43 (1973).

10. Saxena, Ashok, Fibre Sci. and Tech., vol. 12, no. 2, pp. 111-128, (1979).

11. Parhizgar S., Zachary L.W., and Sun C. T., Int. J. Frac., vol. 20, pp. 3-15, (1982). 